several challenges remain, such as device compression, incomplete occlusion, and thromboembolic complications. Changes in intra-aneurysmal flow after the use of the WEB are not well understood and may be important in the performance of the device for aneurysm occlusion. The aim of this study was to characterize the degree of immediate aneurysm occlusion using color-coded quantitative digital subtraction angiography (DSA) after treatment with the WEB.

Materials and Methods We retrospectively reviewed records of 34 patients treated with the WEB between March 2019 and February 2020. Eight patients were excluded from analysis for the use of a concurrent stent or intra-luminal flow-diverter. Measurements of contrast transit times on DSA were calculated with syngo iFlow (Siemens Healthineers AG). Regions of interest were selected within the parent vessel immediately proximal to the aneurysm and within the aneurysm dome before and after treatment with WEB (figure 1). The ratio of aneurysm contrast transit time to parent vessel contrast transit time was obtained before WEB placement and immediately after WEB placement. Transit time ratios were also compared with pre- and post-Pipeline (PED) cases matched for aneurysm size.

Results Out of 26 aneurysms, 24 aneurysms demonstrated an increase in contrast transit time after WEB placement $(2.06 \mathrm{~s}$, $\mathrm{p}<0.05$ ), as measured by iFlow analysis. The ratio of aneurysm-to-parent vessel contrast transit time increased significantly after treatment with WEB $(1.02$ vs. $1.61 ; \mathrm{p}<0.01)$. Similarly, the ratio of contrast transit time increased after PED deployment $(1.07$ vs. $1.51 ; \mathrm{p}<0.01)$, however the ratio increased significantly more after WEB when compared to PED (67\% vs $40 \% ; \mathrm{p}<0.05)$. Average aneurysm size was similar between WEB cases $(n=26)$ and PED cases $(n=26) \quad\left(128.2 \mathrm{~mm}^{3}\right.$ vs $\left.165.1 \mathrm{~mm}^{3}, \mathrm{p}=0.52\right)$. The average number of PED devices used per case was 2.2.

Conclusions High rates of immediate aneurysm contrast stagnation can be achieved with use of the WEB. As a flowdiverting device, the WEB offers a greater degree of immediate aneurysm occlusion than the PED. iFlow analysis provides a quantitative measure of post-treatment effect and could represent a predictive tool for successful long-term occlusion.

Disclosures K. Shah: None. T. White: None. J. Turpin: None. I. Teron: None. J. Katz: None. H. Woo: None.

\section{E-240 SAFETY AND EFFICACY OF TRACSTAR LARGE DISTAL PLATFORM DURING ENDOVASCULAR TREATMENT OF INTRACRANIAL ANEURYSMS}

D Bageac*, R De Leacy. Neurosurgery, The Mount Sinai Hospital, New York, NY

10.1136/neurintsurg-2020-SNIS.271

Introduction Increased procedure time during endovascular treatment of cerebral aneurysms is associated with increased anesthesia related complications and radiation exposure. Elimination of the need for intermediate catheters during aneurysm treatment may decrease procedure time and increase cost effectiveness. A novel device -the TracStar Large Distal Platform (LDP) -may offer more distal final positioning when compared to commonly used guide catheters, thus decreasing the need for guide catheter use. We investigate the safety and efficacy of the TracStar LDP when used during endovascular aneurysm treatment.
Methods We perform a multicenter retrospective review of endovascular cerebral aneurysm embolizations during which the TracStar LDP was utilized. Aneurysm location, procedural information, and complications were recorded as detailed in the operative note. Vascular tortuosity was assessed via pre-procedural CTA. Distal-most position achieved with TracStar was determined by review of intraprocedural imaging.

Results A preliminary analysis of 30 cases was performed. Flow diversion was performed in 25 cases (83\%), and stent assisted coiling was performed in the remainder. The target aneurysm was located in the ICA in 27 cases (90\%), the MCA bifurcation in 2 cases (7\%), and the ACA in 1 case $(3 \%)$. An intermediate catheter was required in 18 cases (60\%). TracStar LDP achieved stable access to the Cavernous ICA in $70 \%$ of cases. Average procedure time was 67 \pm 33 minutes. There were no procedural complications or new neurologic defects after treatment. TracStar LDP was exchanged for an alternative guide catheter in one case due to catheter kinking, and in two cases due to lack of support.

Conclusion Use of Tracstar LDP during flow-diversion and sent-assisted coiling of cerebral aneurysms is safe and effective. Access to the cavernous segment of the ICA was achieved in the majority of cases, and use of an intermediate catheter was not required in $40 \%$ of cases. Final analysis of the the full 60 patient multi-center study will be available for presentation at SNIS Annual 2020.

Disclosures D. Bageac: None. R. De Leacy: None.

\section{E-241 A PROSPECTIVE, MULTICENTER STUDY ASSESSING THE EMBOLIZATION OF INTRACRANIAL ANEURYSMS USING WAVE'M EXTRA SOFT COILS, A PART OF THE PENUMBRA SMART COIL ${ }^{\circledR}$ SYSTEM: STUDY PROTOCOL FOR SURF}

${ }^{1} \mathrm{C}$ Schirmer*, ${ }^{2}$ I Kaminsky, ${ }^{3} \mathrm{M}$ Chaudry, ${ }^{4} \mathrm{O}$ Zaidat, ${ }^{5} \mathrm{~A}$ Alshekhlee, ${ }^{6} \mathrm{P}$ Ramakrishnan, ${ }^{7} \mathrm{M}$ Taqi. ${ }^{1}$ Geisinger, Wilkes-Barre, PA; ${ }^{2}$ Radiology Imaging Associates, Englewood, CO; ${ }^{3}$ Prisma Health, Greenville, SC; ${ }^{4}$ Mercy Health - St. Vincent, Toledo, $\mathrm{OH}_{;}{ }^{5} \mathrm{SSM}$ Health, Bridgeton, MO; ${ }^{6}$ Riverside Regional Medical Center, Newport News, VA; ${ }^{7}$ Los Robles, Thousand Oaks, CA

\subsection{6/neurintsurg-2020-SNIS.272}

Introduction/Purpose Initial clinical evidence has shown that coiling with the SMART COIL ${ }^{\circledR}$ System (Penumbra, Inc.) is a safe and durable treatment option for intracranial aneurysms. ${ }^{1-}$ 5 The WAVE ${ }^{\mathrm{TM}}$ Extra Soft Coil (WAVE) is part of the SMART Coil System and is specifically designed as a fill and finish coil. The primary objective of the SURF study, a post-market registry, is to assess the utility of WAVE as a fill and finish coil to support adequate occlusion at one year follow-up. A secondary objective is to compare the ability of digital subtraction angiography (DSA) and magnetic resonance angiography (MRA) to detect incomplete occlusion in the coiled aneurysms.

Materials and Methods SURF is a post-market, prospective, multicenter, single-arm, observational study that will enroll approximately 800 consecutive patients at up to 50 centers in North America. Patients age $\geq 18$ years, having embolization of intracranial aneurysms, with WAVE as the final finishing coil and Penumbra SMART COIL System accounting for at least $75 \%$ of total number of coils implanted, will be 
included. All subjects will be followed for approximately 1 year. The primary efficacy endpoint is adequate occlusion defined as Raymond-Roy Occlusion Class I and II at final follow-up. The primary safety endpoints are serious adverse events (SAE) within 24 hours post-procedure and devicerelated SAE up to 7 days or discharge. Imaging will be analyzed by an independent core lab to assess aneurysm occlusion rates at follow-up. Imaging modalities include DSA, computed tomography angiography (CTA), and/or MRA. A comparative analysis between imaging modalities will be performed for patients with DSA and MRA.

Results The trial is currently recruiting. Enrollment began November 2019 and the estimated date for study completion is June 2023. Imaging and clinical data collection and core laboratory review are ongoing.

Conclusion We report the design of the SURF study, a postmarket registry that evaluates the safety and performance of the Penumbra SMART COIL System, including WAVE as a fill and finish coil, in the treatment of intracranial aneurysms.

\section{REFERENCES}

1. Daniel B, Henrik S, loannis T, Veit R, Marios-Nikos P. SMART coils for intracranial aneurysm repair - a single center experience. BMC Neurol. 2020 Jan 29;20(1):38.

2. Sokolowski JD, llyas A, Buell TJ, Taylor DG, Chen CJ, Ding D, Raper DMS, Liu KC. SMART coils for intracranial aneurysm embolization: Follow-up outcomes. J Clin Neurosci 2019 Jan;59:93-97.

3. Ilyas A, Buell TJ, Chen CJ, Ding D, Raper DMS, Taylor DG, Sokolowski JD, Liu KC. SMART coils for intracranial aneurysm embolization: Initial outcomes. Clin Neurol Neurosurg 2018 Jan;164:87-91.

4. Spiotta AM, Fargen KM, Lena J, Chaudry I, Turner RD, Turk AS, Huddle D, Loy D, Bellon R, Frei D. Initial Technical Experience with the SMART Coil for the Embolization of Intracranial Aneurysms. World Neurosurg 2017 Jan;97:80-85.

5. Stapleton CJ, Torok CM, Patel AB. Early experience with the Penumbra SMART coil in the endovascular treatment of intracranial aneurysms: Safety and efficacy. Interv Neuroradiol 2016 Dec;22(6):654-658.

Disclosures C. Schirmer: None. I. Kaminsky: None. M. Chaudry: None. O. Zaidat: None. A. Alshekhlee: None. P. Ramakrishnan: None. M. Taqi: None.

\section{Late-Breaking Oral Abstracts}

\section{LB-001 RACIAL DISPARITIES IN ACUTE STROKE THROMBECTOMY MANAGEMENT AND OUTCOMES IN THE UNITED STATES: EVIDENCE FROM THE NVQI-QOD REGISTRY}

\begin{abstract}
${ }^{1} V$ Thirunavu*, ${ }^{2} \mathrm{R}$ Abdalla, ${ }^{2} \mathrm{D}$ Cantrell, ${ }^{3} \mathrm{M}$ Hurley, ${ }^{3} \mathrm{~A}$ Shaibani, ${ }^{1} \mathrm{~B}$ Jahromi, ${ }^{1} \mathrm{M}$ Potts, ${ }^{4} S$ Ansari, ${ }^{5} \mathrm{O}$ NVQI-QOD Registry. ${ }^{1}$ Department of Neurological Surgery, Northwestern University Feinberg School of Medicine, Chicago, IL; ${ }^{2}$ Department of Radiology, Northwestern University Feinberg School of Medicine, Chicago, IL; ${ }^{3}$ Department of Radiology, Department of Neurological Surgery, Northwestern University Feinberg School of Medicine, Chicago, IL; ${ }^{4}$ Department of Radiology, Department of Neurological Surgery, Department of Neurology, Northwestern University Feinberg School of Medicine, Chicago, $I_{i}{ }^{5}$ SNIS, M2S, NPA, AANS, Fairfax, VA
\end{abstract}

\subsection{6/neurintsurg-2020-SNIS.273}

Introduction Endovascular mechanical thrombectomy is the standard of care treatment for acute ischemic stroke secondary to large vessel occlusions, but racial disparities in stroke interventional management and outcomes are not well studied. Moreover, a robust analysis of multiple relevant variables, with consideration of possible confounders, has not been previously conducted. We aimed to evaluate real-world evidence for racial differences in stroke thrombectomy management, short- and long-term outcomes using the Neurovascular Quality Initiative-Quality Outcomes Database (NVQI-QOD) registry.

Methods Data from the NVQI-QOD registry database were analyzed and compared for racial differences with respect to technical and functional outcomes of stroke thrombectomy in 3281 patients from 23 US centers (17 states) between Jan 2015 to March 2020. Race was classified into 4 groups: 1) Caucasian ( $n=2484), 2)$ African American $(n=563), 3)$ Hispanic $(n=109)$, and 4) Asian $(n=105)$. Analysis of variances (ANOVAs), Chi-square tests, Mann Whitney U tests, and multivariate regression models were used to assess racial disparities for 10 outcome variables: final thrombolysis in cerebral infarction (TICI) grade $(n=3182), 24$ hour NIH stroke score (NIHSS) $(\mathrm{n}=2850)$, post-procedure length of stay $(\mathrm{n}=3257), \quad$ ICU days $(\mathrm{n}=2787), \quad$ in-hospital mortality $(\mathrm{n}=3259)$, discharge status $(\mathrm{n}=3281)$, discharge NIHSS $(n=2426)$, discharge modified Rankin score (mRS) $(n=996)$, 90 day re-admission rate $(n=416)$, and 90 day mRS $(n=1184)$. Regression models controlled for demographics, comorbidities, intravenous tPA thrombolysis, and pre-stroke functional measures.

Results ANOVA and Chi-square tests revealed significant differences between racial group means including post-procedure length of stay $(p<0.001)$, ICU days $(p<0.001)$, and inhospital mortality $(\mathrm{p}<0.001)$. There were no significant differences between racial group means for discharge mRS without mortality (African American: 26.7\% favorable outcome, Caucasian: 26.8\%, Hispanic: 27.8\%, Asian: 25\%; $\mathrm{p}=0.90$ ) or for 90 day mRS without mortality (African American: 56.5\% favorable outcome, Caucasian: 51.3\%, Hispanic: $37.5 \%$, Asian: $44.4 \% ; \mathrm{p}=0.54)$. Additional analyses revealed significant differences between African Americans and Caucasians for post-procedure length of stay (mean 10.9 versus 7.9; p<0.001), 24 hour NIHSS (mean 11.2 versus $10.3 ; \mathrm{p}=0.037$ ), ICU days (mean 4.4 versus $3.1 ; \mathrm{p}<0.001)$, and in-hospital mortality $(14.6 \%$ versus $24.5 \% ; \mathrm{p}<0.001)$. Differences between Hispanics and Caucasians were seen for post-procedure length of stay (mean 10.1 versus $7.9 ; \mathrm{p}=0.010$ ), 24-hour NIHSS (mean 12.1 versus 10.3; $\mathrm{p}=0.046$ ), and ICU days (mean 4.3 versus 3.1; $\mathrm{p}=0.011$ ). Differences between Asians and Caucasians were seen for post-procedure length of stay (mean 10.2 versus 7.9; $\mathrm{p}=0.004)$ and ICU days (4.6 versus $3.1 ; \mathrm{p}<0.001)$. Multivariate regression models, with Caucasian set as the reference group, showed higher post-procedure length of stays for African Americans $(p<0.001)$ and Asians $(\mathrm{p}=0.026)$, and higher ICU days for African Americans $(\mathrm{p}<0.001)$ and Asians $(\mathrm{p}=0.003)$.

Conclusion Evidence from the NVQI-QOD registry suggests that there are several racial disparities in stroke thrombectomy management and outcomes, with minorities exhibiting increased post-procedural NIHSS, length of stay, and ICU days. Although African Americans were noted to suffer less in-hospital mortality compared to Caucasians, this did not translate into increased odds of a favorable clinical outcome at 90 days.

Disclosures V. Thirunavu: None. R. Abdalla: None. D. Cantrell: None. M. Hurley: None. A. Shaibani: None. B. Jahromi: None. M. Potts: None. S. Ansari: None. O. NVQIQOD Registry: None. 